\title{
Influence of Massage with Essential Oils to Human Energy
}

\author{
Korotkov Konstantin*1 and Korotkova Anna ${ }^{2}$ \\ ${ }^{1}$ Research Institute of Physical Culture and Sport, Saint-Petersburg, Russia \\ ${ }^{2}$ St Petersburg University of Informational Technologies, Mechanics and Optics, Russia
}

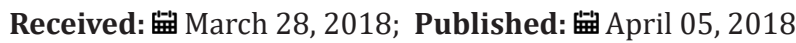

*Corresponding author: Konstantin Korotkov, Research Institute of Physical Culture and Sport, Address: 8 Linia V0 33-11, St Petersburg, Russia; Tel: 79219368394; Email: korotkov2000@gmail.com

\begin{abstract}
Context: Massage is the most common complementary and alternative medicine (CAM) therapy used in hospitals worldwide. As such, it is often the first CAM therapy to be integrated with conventional medicine. However, there are no standard approaches to evaluating the effect of massage to patients mostly taking into account the variety of massage types.
\end{abstract}

Objective: The aim of this study was to develop approach to evaluation the influence of massage with essential oils to the energy and psycho-physiological condition of people.

Design: 83 participants-volunteers took part in the experiments. All experiments were conducted in always the same rooms of the Saint-Petersburg SPA Center (Russia). Specially designed the whole body Energy massage with 7 different types of essential oils and massage without oils have been used. All participants were randomly assigned to 8 groups with 10-11 people in each group. For the evaluation process we have used questioners and Electro photonic Imaging technique (EPI, www.bio-well.com).

Results: Statistically significant changes in EPI parameters and emotional state after massage were found.

Conclusion: Massage has direct influence to the energy resources of the human body and this role of massage treatment is important for the use in CAM medical practice, sport and SPA applications.

Effect of essential oils applications strongly depends on individual preferences, but when selected in accordance with human attention, may increase the influence of massage. Electro photonic technique (EPI/GDV) offers convenient, non-invasive, fast and reliable approach to follow up the energy effect of massage.

Keywords: Energy massage; Aromatherapy; Essential oils; Electro photonic Imaging

\section{Introduction}

The use of massage in complementary and alternative medicine (CAM), spa and wellness applications, and sports treatment is well accepted [1]. Massage has been studied for its effectiveness in the management of chronic pain and for stress and anxiety reduction in cancer patients, hospice patients, and other patient populations. In well populations, massage is typically associated with a reduction in physical and psychological stress [2-7]. In such applications, as in CAM use, aromatherapy may be part of a given massage treatment. Aromatherapy, as a treatment in this

context, integrates the properties of essential oils as both topical and olfactory agents. The effect is systemic, incorporating effects of the aromas directly to the limbic system through inhalation, and through a whole body response to the extended application of the oils to the skin in massage therapy [8]. One of the desired outcomes of these therapies is experiential a subjective perception of well-being. Such an outcome might be defined as a shift towards psychological equilibrium. Techniques for measuring such a result pose an interesting problem. Clinical effects of massage and aromatherapy may be measured by analyzing changes in heart rate, 
blood pressure, salivary cortical levels, and EEG readings [9-13]. Changes in parameters of autonomic nervous system functions may be clinically indicative of effects such as a decrease in stress. However, if well-being is defined in a comprehensive sense, the reduction or absence of stress is a limited measure; well-being is more than simply the absence of something. Furthermore, a sense of well-being is specific to the individual experiencing it, and may vary in its expression within an individual over time.

At the same time there are practically no ways to evaluate the influence of massage by a fast express methods and a lack of standard proven methods. As it is mentioned in this lack of standard policy may impede institutions from offering massage therapy as a clinical service, and may put health care professionals and institutions at risk through a failure to clearly address criteria for practice credentials or malpractice liability.

Wide application of aromatherapy needs as well introduction of fast evaluation approaches for understanding the overall effect of smell and essential oils as a system approach to influence the patient's condition. The technique of Electro photonic Imaging (EPI) allows the recording, from a living subject, of electron and photon emission stimulated by an electromagnetic field, as well as the acquisition of these data by computer image processing. The electric impulse on the camera plate stimulates biological subjects (or chemicals evaporated by this subject) and generates a response in the form of excited gas plasma (that is why in physical terms this approach is known as Gas Discharge Visualization GDV [14]. This plasma emits both light and other electromagnetic fields over a wide frequency band because of the short electrical impulse used (10 microbe). The emissions are directly measured by a chargecoupled device (CCD), the state of the art in measuring low-level light that is used in astrophysics and other scientific endeavors. The CCD registers the pattern of photons detected over time. These digital data are transmitted directly into a computer for data processing, and each image from the light emitted is stored as a graphics file. These two-dimensional images of the light are then used to calculate the area, emission intensity, fracticality, and other parameters. On the basis of the calculated parameters, experimental conclusions are drawn.

Reportedly the EPI has overcome the experimental obstacles of older forms of electro photography. What were previously considered as confounding parameters (such as pressure, finger size, sweating, or changes in physical conductivity) in the older forms of electro photography have been demonstrated by different researchers to be resolved by the new EPI method. Researchers report replicability of findings across different experimenters, different cameras, and different countries [15]. The EPI technique has been found to be effective in evaluating the state of individual human health, in monitoring of individual reactions to different kinds of training [16] and in studying the energy properties of liquids [17-22].
In this paper we are going to present our experimental results of investigation the influence of different types of massage including fragrant essential oils on psycho physiological state on individual.

\section{Participants and Methods}

\section{Subjects}

A total of 83 female and male subjects between the ages of 18 and 66 were recruited in St. Petersburg, Russia, for the study. Participants signed an informed consent form explaining the aims and protocol of the experiments. Subjects were screened for wellness; exclusion criteria included known allergic reactions and conditions which could compromise physical and mental health.

\section{Massage Types}

The massage treatment used in this study was Energy massage, a technique developed specifically to influence the flow of energy in the body. The technique includes deep tissue massage along the erector spinal muscles, and incorporates reflex points relating to the endocrine glands, including a reflexology foot massage. The massage treatment was used with aromatherapy. For the use of aromatherapy with the Energy massage technique, seven aromatic essential oil blends were used. Each blend was formulated with a primary essential oil selected for its therapeutic function in traditional aromatherapy. Essential oil blends were diluted in a professional massage base at a concentration of approximately $1.64 \%$ by volume. The seven sub-groups based on primary essential oils were as follows: Group 1 Amyris Oil, Group 2 Orange Oil, and Group 3 Lavender Oil, Group 4 Palm arosa Oil, Group 5 Grapefruit Oil, Group 6 Geranium Oil, and Group 7 Elemi Oil.

\section{Conditions of the Experiments}

Room was well ventilated; temperature was kept at $72 \pm 2 \mathrm{oF}$, relative humidity not more than $70 \%$. In a single day only one treatment was conducted with particular participant. Before the massage a participant was sitting in a room reading book for accommodation and relaxation. All mobile phones were switched off. Neither participants nor researches were talking during measurements. Before and after the massage participants filled in a special Mood Mapping Evaluation questionnaire.EPI measurements was administered to the panelists before (baseline) and immediately after massage.

Therapists: Therapists were 6 professional masseurs, 4 men, 2 women, Age 29+6. They were trained for a week in Energy Massage [26] and using essential oils by AVEDA trainer Mark Zelic coffer.

Procedure: Participants were assigned to receive an Energy massage administered with one of seven essential oil blends. Control group had same type of massage without oils.

Methods of Study: EPI is an objective test measuring the fingertips All 10 fingers were measured with commercial EPI camera (producer www.bio-well.com) before and immediately after massage. 
Mood Mapping Profile Questionnaire: The Mood Mapping Profile Questionnaire was designed around nine descriptors or adjectives, based on modified Profile of Mood States (POMS) Questioner $[27,28]$ to evaluate mood states of subjects. Subjects were asked to rate how well each of the adjectives described their current state of feeling on a 5-point scale of intensity from "not at all" (1) to "extremely" (5). Subjects completed identical scales before and after treatment.

\section{Data Processing}

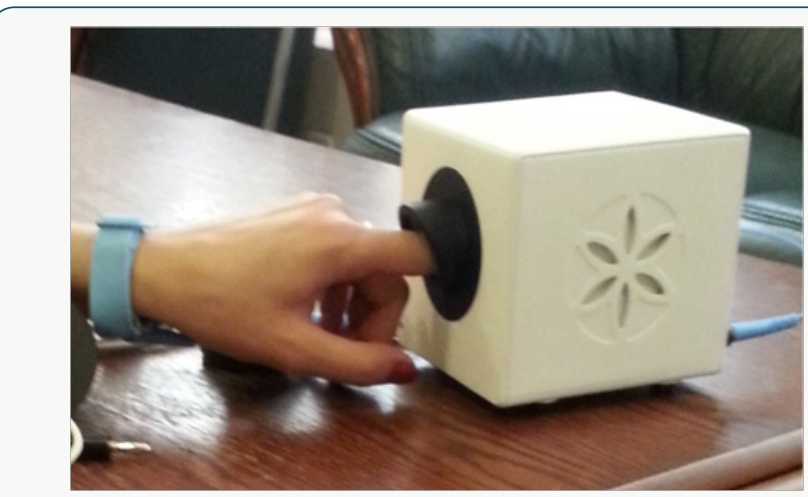

Figure 1: Capturing images with Bio-Well GDV device.

Pre-processing of data was handled by software suites that had been especially developed to interact with the EPI equipment (Figure1). This software cleans the background from the image of glow around human finger (Figure 2) using the following algorithm: all image points removed by noise filtration are shown as white background; then the image is divided into related fragments, neighboring pixels adjacent to each other vertically or horizontally, is considered a portion of the image. Fragments are removed in which the area is less than 30 pixels. As a result, we get a filtered image. For visual estimation of the image, there is an algorithm of pseudo-coloring. The brightest glow points are colored in the shades of blue, less bright points are colored in the shades of red. Points are colored in yellow when the intensity is higher than the noise level, but lower than the base noise level for the given frame. Software calculates a set of parameters with image processing technique. In this study we calculated the Area of an image number of pixels with intensity higher than the background level; Intensity or Brightness, ranged in software from 0 to 255; as well as Stress

Table 1: Averaged on the group Area in pixels of an EPI images and Stress Index "Before" and "After" the massage for different groups.

\begin{tabular}{|c|c|c|c|c|c|c|c|c|c|}
\hline \multirow{2}{*}{$\begin{array}{c}\text { EPI } \\
\text { parameters }\end{array}$} & Group & Oil 1 & Oil 2 & Oil 3 & Oil 4 & Oil 5 & Oil 6 & Oil 7 & Massage Without Oils \\
\hline & Group size & 10 & 10 & 10 & 11 & 11 & 10 & 10 & 10 \\
\hline \multirow{3}{*}{ Area } & Before & 7939 & 9188 & 6821 & 7987 & 8239 & 8080 & 7439 & 7235 \\
\hline & After & 10727 & 10888 & 10567 & 10196 & 10910 & 9489 & 11875 & 8489 \\
\hline & $\mathrm{p}$ value & $<0.01$ & $<0.01$ & $<0.001$ & $<0.001$ & $<0.01$ & $<0.01$ & $<0.001$ & $<0.05$ \\
\hline \multirow{3}{*}{ Stress Index } & Before & 2.4 & 3.6 & 2.8 & 2.9 & 3.3 & 3.8 & 3.4 & 3.6 \\
\hline & After & 1.6 & 1.8 & 2.0 & 1.4 & 2.1 & 2.2 & 1.9 & 2.9 \\
\hline & $\mathrm{p}$ value & $<0.005$ & $<0.01$ & $<0.007$ & $<0.03$ & $<0.04$ & $<0.001$ & $<0.003$ & 0.08 \\
\hline
\end{tabular}


Table 2: Comparison between before-after massage treatment for different mood state results for the eight groups testes in the study.

\begin{tabular}{|c|c|c|c|c|c|c|c|c|c|}
\hline \multirow{2}{*}{ Mood state } & Group & Oil 1 & Oil 2 & Oil 3 & Oil 4 & Oil 5 & Oil 6 & Oil 7 & Massage Without Oils \\
\hline & Group size & 10 & 10 & 10 & 11 & 11 & 10 & 10 & 10 \\
\hline \multirow{3}{*}{ Grounded } & Before & 1.3 & 2.1 & 2.0 & 2.9 & 2.0 & 2.7 & 2.3 & 2.5 \\
\hline & After & 1.6 & 1.4 & 1.3 & 1.7 & 2.1 & 2.3 & 1.7 & 2.3 \\
\hline & $\mathrm{p}$ value & 0.6 & 0.2 & 0.03 & 0.001 & 0.7 & 0.3 & 0.1 & 0.8 \\
\hline \multirow{3}{*}{ Inspired } & Before & 2.2 & 2.5 & 2.6 & 1.6 & 2.2 & 1.5 & 2.1 & 2.3 \\
\hline & After & 3.3 & 3.1 & 2.7 & 2.5 & 2.6 & 2.7 & 2.3 & 2.3 \\
\hline & $\mathrm{p}$ value & 0.2 & 0.5 & 0.1 & 0.001 & 0.2 & 0.02 & 0.3 & 0.9 \\
\hline \multirow{3}{*}{ Energized } & Before & 1.6 & 2.7 & 1.7 & 2.2 & 2.1 & 2.6 & 2.3 & 2.4 \\
\hline & After & 1.7 & 1.6 & 1.3 & 1.8 & 1.1 & 1.7 & 1.6 & 1.8 \\
\hline & $\mathrm{p}$ value & 0.5 & 0.02 & 0.4 & 0.3 & 0.2 & 0.04 & 0.5 & 0.06 \\
\hline \multirow{3}{*}{ Balanced } & Before & 2.8 & 1.7 & 2.7 & 2.0 & 2.2 & 2.2 & 2.0 & 2.6 \\
\hline & After & 3.3 & 3.2 & 3.2 & 3.2 & 3.5 & 3.4 & 3.0 & 2.9 \\
\hline & $\mathrm{p}$ value & 0.3 & 0.01 & 0.09 & 0.001 & 0.001 & 0.002 & 0.04 & 0.07 \\
\hline \multirow{3}{*}{ Creative } & Before & 2.2 & 2.9 & 2.5 & 2.0 & 1.7 & 2.2 & 1.6 & 1.8 \\
\hline & After & 1.8 & 3.0 & 2.8 & 2.0 & 3.0 & 2.4 & 2.5 & 2.1 \\
\hline & $\mathrm{p}$ value & 0.4 & 0.4 & 0.1 & 0.1 & 0.02 & 0.6 & 0.03 & 0.06 \\
\hline \multirow{3}{*}{ Focused } & Before & 2.1 & 2.4 & 2.5 & 2.3 & 2.2 & 1.5 & 2.4 & 2.6 \\
\hline & After & 1.7 & 2.1 & 2.5 & 2.4 & 2.2 & 2.1 & 2.2 & 2.5 \\
\hline & $\mathrm{p}$ value & 0.6 & 0.2 & 1 & 0.8 & 1 & 0.3 & 0.6 & 0.9 \\
\hline \multirow{3}{*}{ Aware } & Before & 2.5 & 2.2 & 2.9 & 2.6 & 2.4 & 2.5 & 2.6 & 2.6 \\
\hline & After & 1.3 & 1.8 & 2.2 & 2.4 & 2.1 & 2.3 & 2.2 & 2.7 \\
\hline & $\mathrm{p}$ value & 0.003 & 0.2 & 0.06 & 0.6 & 0.6 & 0.5 & 0.4 & 0.9 \\
\hline \multirow{3}{*}{ Relaxed } & Before & 1.6 & 1.5 & 2.1 & 2.2 & 1.5 & 1.3 & 1.7 & 2.4 \\
\hline & After & 3.5 & 4.1 & 3.9 & 1.8 & 3.5 & 3.6 & 3.6 & 3.2 \\
\hline & $\mathrm{p}$ value & 0.002 & 0.001 & 0.001 & 0.2 & 0.001 & 0.001 & 0.001 & 0.05 \\
\hline \multirow{3}{*}{ Peaceful } & Before & 3.5 & 3.3 & 3.7 & 3.1 & 3.8 & 2.4 & 3.1 & $2 / 3$ \\
\hline & After & 4.1 & 3.0 & 4.2 & 4.3 & 4.2 & 3.7 & 3.8 & 3.3 \\
\hline & $\mathrm{p}$ value & 0.001 & 0.02 & 0.001 & 0.001 & 0.2 & 0.01 & 0.002 & 0.03 \\
\hline
\end{tabular}

\section{Discussion}

As was shown in a lot of medical research works on Electro photonics, increase of Area may be considered as a positive outcome of treatment [23-28]. As we see from Table 1, this effect was registered as statistically significant for most of the panelists having massage both with and without essential oils. From the presented data we may see that response to massage was quite different between panelists. In mood-mapping questionnaire they all mentioned "relaxing", "calming" and "balancing" effects of massage. Which means that subjective feeling may be quite tricking and different from the real psychophysiological effect? For massage without oils the level of psychological response was less compared with massage using oils.

This shows that comparison between different types of massage without objective measurements is practically impossible. As we see from graphs (Figure 3) the highest effect was registered from the massage with essential Oils 1, 3 and 7 (Amyris Oil, Lavender Oil and Elemi Oil); for the massage with essential Oils 4 and 5 (Palm arosa
Oil and Grape fruit Oil) the effect was quite strong as well, while for the Oils 2 and 6 (Orange Oil and Geranium Oil) the effect was compatible with massage without oils. These results demonstrate that the effect of essential oils is very subjective and selection of the oils should be done based on individual preferences. Not to mention that some people have allergy to smell. We do not know whether related to the preferences of the particular participants, or has more common meaning. At the same time this selection would have been impossible to determine from the subjective experience.

Measured response from the right hand was stronger than from the left. (In EPI technology measurements is taken from 10 fingers of both hands). The right hand is related to the left part of the brain. This part is responsible for logic, speech, control of behavior and physical functions; as opposed to the right part of the brain which is responsible for emotions and subconscious processing of information. So we may conclude that response to massage was more due to the physiological processes than to emotional. What do all these changes mean? It is known that electron-excited states 
in complex molecular systems are the main reservoir of free energy in biological processes. These excited states are continuously supported at the expense of electron circulation in the biosphere. The main "working substance" is water and the energy source is the sun. Stimulated opto-electron emission in the EPI process is determined mainly by the transport of delocalized $\pi$-electrons, realized in electrically non-conducting tissue by way of the quantum electron tunnel mechanism. This proposition allows an assumption that the EPI/GDV technique provides indirect judgment about the level of energy resources at the molecular level of functioning in structural-protein complexes. So we may conclude that measured responses characterize the changes of the energy resources of the body.

\section{Conclusion}

Based on the presented results we can make several conclusions:

a) Massage has direct influence to the energy resources of the human body and this role of massage treatment is important for the use in CAM medical practice, sport and SPA applications.

b) Effect of essential oils applications strongly depends on individual preferences, but when selected in accordance with human attention may increase the influence of massage.

c) Electro photonic technique (EPI/GDV) offers convenient, non-invasive, fast and reliable approach to follow up the energy effect of massage.

\section{Limitations and The Potential Future Studies}

In the future studies we plan to investigate correlation between individual response to massage, gender, age and psychological profile of the participants. This would help to select individual type of aromas for people before the application of massage.

\section{References}

1. Myklebust M, Iler J (2007) Policy for therapeutic massage in an academic health center: a model for standard policy development. J Altern Complement Med 13: 471-475.

2. Dawson LG, Dawson KA, Tiidus PM (2004) Evaluating the influence of massage on leg strength, swelling, and pain follows a half marathon. J Sports Science Medicine 3: 37-41.

3. Plews Ogan M, Owens JE, Goodman M (2005) A pilot study for evaluating mindfulness-based stress reduction and massage for the management of chronic pain. J Gen Intern Med 20(12): 1136-1138.

4. Wardle JL, Barnett R, Adams J (2015) Practice and research in Australian massage therapy: a national workforce survey. Int J Ther Massage Bodywork 8(2): 2-11.

5. Resnic PB (2016) Comparing the Effects of Rest and Massage on Return to Homeostasis Following Submaximal Aerobic Exercise: a Case Study. Int J Ther Massage Bodywork 9(1): 4-10.

6. Porcino AJ, Verhoef MJ (2010) The Use of Mixed Methods for Therapeutic Massage Research. Int J Ther Massage Bodywork 3(1): 15-25.

7. Mustafa K, Furmanek MP, Knapik A, Bacik B, Juras G (2015) The Impact of the Swedish massage on the Kinesthetic Differentiation in Healthy Individuals. Int J Ther Massage Bodywork 8(1): 2-11.
8. Shi Ming Peng, Malcolm Koo, Zer-Ran Yu (2009) Effects of Music and essential Oil Inhalation on Cardiac Autonomic Balance in Healthy Individuals. J of Alternative Complementary Medicine 15(1): 53-57.

9. Soden K, Vincent K, Craske S (2004) A randomized controlled trial of aromatherapy massage in a hospicesetting. Palliat Med 18: 87-92.

10. Rho K, Han S, Kim K (2006) Effects of aromatherapy massage on anxiety and self-esteem in Korean elderly women: a pilotstudy. Intern J Neuroscience 116(2): 1447-1455.

11. Cambron JA, Dexheimer J, Coe P (2006) Changes in blood pressure after various forms of therapeutic massage: a preliminary study. J Altern Complement Med 12(1): 65-70.

12. Hongratanaworakit T, Buchbauer G (2006) Relaxing effect of ylangy langoilon humans after transdermal absorption. Phytother Res 20(9): 758-763.

13. Diego MA, Field T, Sanders C (2004) Massage therapy of moderate and light pressure and vibrator effects on EEG and heartrate. Intern J Neuroscience 114(1): 31-45.

14. Shiva KK, Srinivasan TM, Nagendra HR, Marimuthu P (2016) Electrophotonic Imaging Based Analysis of Diabetes. Int J of Altern and Complement Medicine 4(5): 134-137.

15. Bhargav H, Srinivasan TM, Varambally S, Gangadhar BN, Koka P (2015) Effect of mobile phone induced electromagnetic field on brain haemodynamics and human stem cell functioning possible mechanism link to cancer risk and early diagnostic values of electrophotonic imaging. J Stem cells 10(4): 287-294.

16. Yakovleva EG, Buntseva OA, Belonosov SS, Feorov ED, Zarubina TV (2015) Identifying Patients with Colon Neoplasias with Gas Discharge Visualization Technique. J of Alternative Complementary Medicine 21(11): 720-724.

17. Gagua PO, Gedevanishvili E, Georgobiani L, Kapanadze A, Korotkov KG, et al. (2004) Experimental study of the GDV technique application in oncology. In: Measuring Energy Fields, Korotkov K (edn.); Fair Lawn: Backbone Publishing 43-50.

18. Bell I, Lewis DA, Brooks AJ, Lewis SE, Schwartz GE (2003) Gas Discharge Visualization Evaluation of Ultramolecular Doses of Homeopathic Medicines Under Blinded, Controlled Conditions. J of Alternative and Complementary Medicine 9(1): 25-38.

19. Korotkov K, Krizhanovsky E, Borisova M, Hayes M, Matravers P, et al. (2004) The Research of the Time Dynamics of the Gas Discharge Around Drops of Liquids. J of Applied Physics 95(7): 3334-3338.

20. Giacomoni P, Hayes M, Korotkov K, Krizhanovsky E, Matravers P, et al. (2003) Investigation of Essential Oils and Synthetic Fragrances using the Dynamic Gas Discharge Visualization Technique. World Perfume Congress, Seoul Korea p. 18.

21. McNair DM, Heuchert JWP, Shillony E (2001) Research with the Profile of Mood States (POMS) 1964-2002: A comprehensive bibliography: MultiHealth Systems. Toronto, Canada.

22. McNair DM, Lorr M, Droppleman LF (1971) Manual for the Profile of Mood States: Educational and Industrial Testing Services. San Diego, Canada.

23. Korotkov K, Williams B, Wisneski L (2004) Biophysical Energy Transfer Mechanisms in Living Systems: The Basis of Life Processes. J of Alternative and Complementary Medicine 10(1): 49-57.

24. Korotkov K (2017) The Energy of Health. Amazon Publishing.

25. Korotkov KG, Orlov DV, Williams BO (2010) Application of Electro photon Capture (EPI) Analysis Based on Gas Discharge Visualization (GDV) Technique in Medicine: A Systematic Review. J of Alternative and Complementary Medicine 16(1): 13-25. 
26. Kostyuk N, Meghanathan N, Isokpehi RD, Bell T, Rajnarayanan R (2010) Biometric Evaluation of Anxiety in Learning English as a Second Language. International Journal of Computer Science and Network Security 10(1): 220-229.

27. Polushin J, Levshankov A, Shirokov D, Korotkov K (2009) Monitoring Energy Levels during treatment with GDV Technique. J of Science of Healing Outcome 2(5): 5-15.
28. Rgeusskaja GV, Listopadov UI (2009) Medical Technology of Electro photonics. Gas Discharge Visualization in Evaluation of Cognitive Functions. J of Science of Healing Outcome 2(5): 16-19.

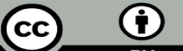

This work is licensed under Creative Commons Attribution 4.0 License

To Submit Your Article Click Here:

Submit Article

DOI: $10.32474 /$ OAJBEB.2018.02.000131

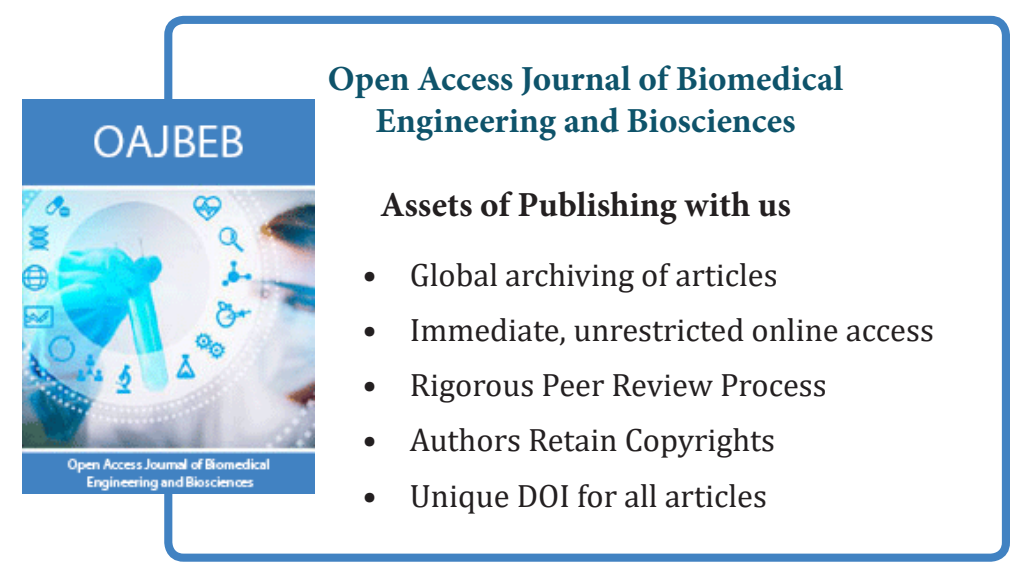

